Journal Club

Editor's Note: These short, critical reviews of recent papers in the Journal, written exclusively by graduate students or postdoctoral fellows, are intended to summarize the important findings of the paper and provide additional insight and commentary. For more information on the format and purpose of the Journal Club, please see http://www.jneurosci.org/misc/ifa_features.shtml.

\title{
Cell Adhesion Complexes Localize Presynaptic Vesicles by Regulating Local Actin Polymerization
}

\author{
Elliott B. Merriam ${ }^{\star}$ and Xindao $\mathrm{Hu}^{*}$ \\ Neuroscience Training Program, University of Wisconsin-Madison, Madison, Wisconsin 53706 \\ Review of Sun and Bamji
}

In the developing nervous system, synapse formation becomes possible when outgrowing axons and dendrites come into contact with one another. In order for functional synaptic connections to be established, however, both presynaptic and postsynaptic protein complexes must be recruited and stabilized at appropriate points of cell-cell contact (McAllister, 2007). Synaptic proteins are thought to mobilize within developing axons and dendrites long before synaptogenesis occurs, with the recruitment of synaptic vesicles occurring within minutes to hours after the initiation of axodendritic contact. This process is thought to be regulated at least in part by adhesion molecules and their intracellular binding partners.

Transsynaptic adhesion molecules forming the cadherin/ $\beta$-catenin/scribble complex regulate clustering of synaptic vesicles (SVs) in axons (Bamji et al., 2003; Sun et al., 2009). SV clustering also depends on filamentous actin, which is enriched at synapses (Colicos et al., 2001; Zhang and Benson, 2001), and actin depolymerizing drug decreases SV mobility (Sabo et al., 2006). However, previous

Received Jan. 12, 2012; revised Feb. 6, 2012; accepted Feb. 8, 2012.

We thank Dr. Erik Dent and The Dent Lab for their continual support of our intellectual development.

*E.B.M. and X.H. contributed equally to this work.

Correspondence should be addressed to either Elliott B. Merriam or

Xindao Hu, 340 Bardeen Labs, 1300 University Avenue, Madison, WI 53706.

E-mail: ebmerriam@wisc.edu or xhu7@wisc.edu.

DOI:10.1523/JNEUROSCI.0191-12.2012

Copyright $\odot 2012$ the authors $\quad 0270-6474 / 12 / 323955-03 \$ 15.00 / 0$ studies have not defined the precise role of actin polymerization in SV clustering or localization. Furthermore, relatively little is known about how actin dynamics are regulated at sites of synaptic contact to maintain proper localization of synaptic materials. In a recent study in The Journal of Neuroscience, Sun and Bamji (2011) shed light on these questions by demonstrating a direct role for F-actin in active zone formation. They also provided strong evidence that the Rac/Cdc42-specific guanine nucleotide exchange factor (GEF) $\beta$-pix localizes to sites of cell-cell contact through a direct interaction with cadherin/ $\beta$-catenin/scribble, and it promotes SV clustering by increasing actin polymerization at these sites (Sun and Bamji, 2011).

Previous studies suggested that F-actin is important for SV clustering because clustering was reduced after actin polymerization was disrupted. Sun and Bamji (2011) took this one step further by increasing F-actin polymer content through two distinct approaches. First, they found that an inhibitor of calpain, which normally suppresses actin polymerization, increased both the density and size of actin puncta in the axon. The density and size of SV clusters associated with these actin puncta also increased, but the size and density of clusters not associated with actin was unchanged, suggesting that increased F-actin content is responsible for the increased SV clustering. Second, the authors overexpressed cortactin in hippocampal neurons. Cortactin stabilizes Arp2/3 complexes, thus enhancing actin polymerization. In addition to providing a distinct cellular mechanism for increasing F-actin, this manipulation targeted only a subset of cells, thus eliminating potentially confounding non-cell-autonomous effects that might be expected from bath application of a drug. Cortactin overexpression, like calpain inhibition, increased the density and size of actin puncta and SV clusters associated with actin puncta, but not of SV clusters that were not associated with actin. Live-imaging experiments revealed that the density of stable, rather than mobile, SV clusters was specifically enhanced by cortactin overexpression. Importantly, the number of actin/SV puncta associated with postsynaptic markers was unchanged by either cortactin overexpression or calpain inhibition. These results provide direct evidence for a positive role of F-actin in clustering of SVs, but suggest that actin polymer is not sufficient for appropriate localization of SV clusters at presynaptic sites.

How, then, are SV clusters localized to sites of nascent synaptic contact to form stable presynaptic specializations? To address this question, Sun and Bamji (2011) built off of their previous work implicating the cadherin $/ \beta$-catenin/scribble complex in SV localization at synaptic sites (Bamji et al., 2003; Sun et al., 2009), and turned their attention toward $\beta$-pix, a Rac/Cdc42-specific GEF that forms a complex with scribble and can promote actin polymerization via its GEF activity (Audebert et al., 2004). First, Sun and Bamji (2011) established that $\beta$-pix localizes to presynaptic terminals by visualizing immunolabeled or fluorescently 
tagged $\beta$-pix with immunolabeled presynaptic and postsynaptic markers. They also confirmed that $\beta$-pix interacts with the cadherin/ $\beta$-catenin/scribble complex at synapses by performing coimmunoprecipitation assays on synaptosomal fractions. Importantly, by using shRNA-mediated knockdown of scribble and N-cadherin, the authors show that these proteins are required for normal synaptic localization of presynaptic $\beta$-pix.

Does $\beta$-pix contribute to actin polymerization and SV clustering at presynaptic sites? To test this, Sun and Bamji (2011) knocked down $\beta$-pix expression and expressed a dominant-negative $\beta$-pix construct, and found that reducing either $\beta$-pix expression or GEF activity decreased both the size of presynaptic actin puncta and dramatically reduced SV clustering. Importantly, although clustering of synaptophysin-positive SVs was reduced when $\beta$-pix activity was impaired, clustering of other presynaptic and postsynaptic proteins (bassoon and PSD-95) into discrete puncta remained normal. Sun and Bamji (2011) conclude the study by showing that cortactin overexpression fully rescues the loss of SV clustering caused by $\beta$-pix knockdown, indicating that it is specifically $\beta$-pix's ability to promote actin polymerization that is essential for presynaptic SV clustering.

Together, Sun and Bamji's (2011) findings strongly suggest that in axons, $\beta$-pix aggregates at sites of cell-cell contact through an interaction with cadherin/ $\beta$-catenin/ scribble, and that $\beta$-pix activity at these sites locally promotes actin polymerization, resulting in F-actin-dependent capture of SVs. This study is significant because it identifies a component of synaptic adhesion complexes that directly alters the local actin cytoskeleton to trap SVs. In this way, the work of Sun and Bamji (2011) successfully bridges a crucial gap in our understanding of the relationship between cell-cell interactions and regulation of molecular transport during synaptogenesis.

A recently published study by Waites et al. (2011) also examined the role of actin in assembly of presynaptic specializations and offers findings that complement those of Sun and Bamji (2011). Waites et al. (2011) found that the active zone protein Piccolo is essential for both F-actin assembly and calcium/calmodulin-dependent protein kinase II (CaMKII) recruitment at presynaptic sites. Using shRNA-mediated knockdown of Piccolo in dissociated hippocampal neurons, Waites et al. (2011) demonstrated that loss of Piccolo impaired presynaptic actin assembly and CaMKII clustering while increasing SV release. Importantly, these Piccolo knockdown phenotypes were rescued by treatment with the actin-stabilizing drug jasplakinolide and were copied by knockdown of Profilin2, which promotes actin polymerization.

Together, the results presented by Sun and Bamji (2011) and by Waites et al. (2011) indicate that actin organization serves multiple regulatory functions at presynaptic release sites. Interestingly, dense-core Piccolo transport vesicles (PTVs) are thought to be recruited to presynaptic sites either at the same time or immediately following clustering of SVs (McAllister, 2007). It is therefore possible that during synaptogenesis, PTVs are targeted to sites of nascent synaptic contact through an F-actin-dependent trapping mechanism similar or identical to that which regulates SV localization. Future experiments combining targeted manipulations of F-actin assembly with visualization of specific types of vesicles could be performed to test whether this is true.

Many of the mechanisms for transport and localization of presynaptic materials remain poorly understood. In developing axons, bundled microtubules are enriched throughout the axon shaft, whereas actin filaments concentrate at discrete presynaptic sites. Live-cell imaging studies have shown that both PTVs and SVs are highly mobile within axons and travel at a velocity of 0.1-1 $\mu \mathrm{m} / \mathrm{s}$ (McAllister, 2007), suggesting that they are primarily transported by microtubule-associated motor proteins. Among the kinesin superfamily proteins, three anterograde microtubule motor proteins have been shown to facilitate SV protein trafficking: the conventional kinesin-1 (Cai et al., 2007), KIF1A, and KIF1B- $\beta 2$ (McAllister, 2007). Interestingly, the actinassociated motor protein myosin $\mathrm{Va}$ was found to interact directly with the rod domain of mouse ubiquitous kinesin heavy chain in a yeast two-hybrid screen (Huang et al., 1999). Furthermore, transport of myosin Va-labeled vesicles was impaired by disruption of the microtubule network in neurons with nocodazole, whereas lactrunculin did not dampen myosin Va-labeled vesicle movement, indicating that microtubule-based motors are responsible for trafficking of vesicles that contain myosin Va (Bridgman, 1999). It is possible that when these vesicles encounter high levels of F-actin, such as at sites of high $\beta$-pix activity, they detach from microtubules and attach to actin filaments through myosin Va. Such a mechanism could account for localization of presynaptic materials at sites of cell-cell adhesion, but this possibility remains untested.

Appropriate targeting of synaptic machinery to sites of axodendritic contact likely involves a complex interplay between the neuronal cytoskeleton, molecular motors, and the vesicular cargos they carry. While the studies of Sun and Bamji (2011) and Waites et al. (2011) shed significant light on the relationship between cell adhesion complexes, local actin organization, and vesicle transport, these studies do not evaluate the relative contributions of actin- or microtubule-associated motor proteins. Future experiments should ultimately seek to manipulate specific motor proteins to determine their respective contributions in vesicle transport and localization. This is experimentally challenging because of the tremendous diversity of motor proteins and their high potential for functional redundancy in mammalian neurons (Hirokawa et al., 2010), and because of the lack of tools available for manipulating specific proteins' functions with great temporal and spatial control in situ. Recent technical developments, such as optical control of molecular activity (Bacchus and Fussenegger, 2011), may ultimately offer a solution to these fundamental challenges and enable neurobiologists to decipher the precise contributions of cell-cell adhesion complexes, motor proteins, and cytoskeletal organization in synaptogenesis.

\section{References}

Audebert S, Navarro C, Nourry C, Chasserot-Golaz S, Lécine P, Bellaiche Y, Dupont JL, Premont RT, Sempéré C, Strub JM, Van Dorsselaer A, Vitale N, Borg JP (2004) Mammalian scribble forms a tight complex with the $\beta$-pix exchange factor. Curr Biol 14:987-995.

Bacchus W, Fussenegger M (2011) The use of light for engineered control and reprogramming of cellular functions. Curr Opin Biotechnol. Advance online publication. Retrieved January 7 , 2012. doi:10.1016/j.copbio.2011.12.004.

Bamji SX, Shimazu K, Kimes N, Huelsken J, Birchmeier W, Lu B, Reichardt LF (2003) Role of beta-catenin in synaptic vesicle localization and pre-synaptic assembly. Neuron 40:719-731.

Bridgman PC (1999) Myosin Va movement in normal and dilute-lethal axons provide support for a dual filament motor complex. J Cell Biol 146:1045-1060.

Cai Q, Pan PY, Sheng ZH (2007) Syntabulinkinesin-1 family member 5B-mediated axonal transport contributes to activity-dependent presynaptic assembly. J Neurosci 27:7284-7296

Colicos MA, Collins BE, Sailor MJ, Goda Y (2001) Remodeling of synaptic actin induced by photoconductive stimulation. Cell 107:605-616. 
Hirokawa N, Niwa S, Tanaka Y (2010) Molecular motors in neurons: transport mechanisms and roles in brain function, development and disease. Neuron 68:610-638.

Huang JD, Brady ST, Richards BW, Stenolen D, Resau JH, Copeland NG, Jenkins NA (1999) Direct interaction of microtubule and actin based transport motors. Nature 397:267270.

McAllister AK (2007) Dynamic aspects of CNS synapse formation. Annu Rev Neurosci 30:425-450.

Sabo SL, Gomes RA, McAllister AK (2006) Formation of presynaptic terminals at predefined sites along axons. J Neurosci 26:10813-10825.

Sun Y, Bamji SX (2011) $\beta$-pix modulates actinmediated recruitment of synaptic vesicles to synapses. J Neurosci 31:17123-17133.

Sun Y, Aiga M, Yoshida E, Humbert PO, Bamji SX
(2009) Scribble interacts with beta-catenin to localize synaptic vesicles to synapses. Mol Biol Cell 20:3390-3400.

Waites CL, Leal-Ortiz SA, Andlauer TF, Sigrist SJ, Garner CC (2011) Piccolo regulates the dynamic assembly of presynaptic f-actin. J Neurosci 31:14250-14263.

Zhang W, Benson DL (2001) Stages of synapse development defined by dependence of F-actin. J Neurosci 21:5169-5181. 